\title{
A SINGULAR BOUNDARY VALUE PROBLEM FOR NEUTRAL EQUATIONS
}

\author{
VASIL G. ANGELOV \\ Academy of Medicine, Sofia \\ 1184 Sofia, P.0. Box 37, \\ Bulgaria
}

(Received June 21, 1983)

ABSTRACT. Using some previous results of the author and Kirk-Schbneberg, theorems for the existence and uniqueness of an absolutely continuous solution of a singular boundary value problem for neutral equations have been proved.

KEY WORDS AND PHRASES. Neutral equations, boundary value problem, singular.

1980 MATHEMATICS SUBJECT CLASSIFICATION CODE. $34 K 10$.

\section{INTRODUCTION.}

The aim of the present paper is to obtain conditions for the existence and uniqueness of an absolutely continuous solution of a singular boundary value problem for neutral equations, using the results due to Angelov [1] and Kirk-Schơneberg [2].

Let us state the singular boundary value problem: we look for an absolutely continuous solution $y(t)$ of the initial value problem

$$
\begin{aligned}
y^{\prime}(t) & =x\left(\mu, t,\left[y\left(\Delta_{i}(t)\right)\right]_{i=1}^{m},\left[y^{\prime}\left(\tau_{1}(t)\right)\right]_{1=1}^{n}\right), t>0 \\
y(t) & =\psi(t, \mu), y^{\prime}(t)=\frac{\partial \psi(t, \mu)}{\partial t}, t<0
\end{aligned}
$$

which satisfies the condition $\lim _{t \rightarrow \infty} y(t)=y_{\infty}$, where $y_{\infty}$ is given. Here the unknown function $y(t)$ and the parameter $\mu$ take values in some Banach space $B$ with a norm $\|\cdot\|$ and

$$
\begin{aligned}
& {\left[y\left(\Delta_{i}(t)\right)\right]_{i=1}^{m}=\left(y\left(\Delta_{1}(t)\right), \ldots, y\left(\Delta_{m}(t)\right)\right),} \\
& {\left[y^{\prime}\left(\tau_{1}(t)\right)\right]_{1=1}^{n}=\left(y^{\prime}\left(\tau_{1}(t)\right), \ldots, y^{\prime}\left(\tau_{n}(t)\right)\right) .} \\
& \text { We shall use the standard denotations: } \\
& R^{1}=(-\infty, \infty) ; R_{+}^{1}=[0, \infty) ; R_{-}^{1}=(-\infty, 0] .
\end{aligned}
$$


Let us set $x(t)=y^{\prime}(t)$ for $t>0$ and $\varphi(t, \mu)=\frac{\partial \psi(t, \mu)}{\partial t}$ for $t<0$. Then we have

$$
\begin{aligned}
& x(t)=x\left(\mu, t,\left[\psi(0, \mu)+\int_{0}^{\Delta_{i}(t)} x(s) d s\right]_{i=1}^{m},\left[x\left(\tau_{1}(t)\right)\right]_{1=1}^{n}\right), t>0 \\
& x(t)=\varphi(t, \mu), t \leqslant 0 .
\end{aligned}
$$

The integrals are in a Bochner's sense. (Hille-Phillips [3]).

The method used by Angelov [1] is not immediately applicable to the singular boundary value problem for an equation(1.1). In order to illustrate this fact we shall consider the following particular case:

$$
y^{\prime}(t)=A(t) \mu+X\left(\mu, t,\left[y\left(\Delta_{i}(t)\right)\right]_{i=1}^{m},\left[y^{\prime}\left(\tau_{1}(t)\right)\right]_{1=1}^{n}\right)
$$

which occurs in the applications. Here $A(t)$ is a family of linear continuous operators. We must seek a solution of $(1.3)$ in the space $L^{1}\left(R^{1}, B\right)$ which implies $\|A(t) \mu\| L^{1}\left(R_{+}^{1}\right)$. But then the supposition for the existence of $A^{-1}(t)$, (Seidov [4]), is not natural, because one of the conditions for the existence of an inverse operator is $\|A(t) \mu\|>m\|\mu\|$ (Kantorovich-Akilov [5], p. 209).

That is why we shall apply some recent results of Kirk-Schbneberg [2] for the operator equation in $\mu$.

Let us formulate some auxiliary propositions due to Angelov [1]. If $B_{i}(i=1,2)$ are Banach spaces with norms $\|.\|_{i}$, then:

PROPOSITON 1 [1]. Let the following conditions hold:

1. The nonlinear continuous operators $N_{i}: B_{i} \times B \rightarrow B_{i}$ satisfy the inequalities

$\|\left((1+\lambda \gamma-\lambda \alpha) x+\lambda N_{i}(x, \mu)\right)-\left((1+\lambda \gamma=\lambda \alpha) y+\lambda N_{i}(y, \mu)\left\|_{i} \geqslant\right\| x-y \|_{i}(i=1,2)\right.$ for every $x, y e B_{i}, \lambda>0$, $\mu$ e $B$, for some $\gamma>0, \alpha>0$.

2. RESULTS.

The linear map $j: B_{1} \rightarrow B_{2}$ satisfies the condition $j\left(N_{1}(x, \mu)\right)=$ $=\mathrm{N}_{2}(j x, \mu)$ for every $x \in B_{1}$ and $\mu \in B$.

Then for maps $x(\mu): B \rightarrow B_{1}$ and $y(\mu): B+B_{2}$ connected by the cond $i-$ tion $\mathrm{jx}(\mu)=\mathrm{N}_{2}(y(\mu), \mu)+\gamma y(\mu)$ there exists a unique map $z(\mu): B+B_{1}$ for which $N_{1}(z(\mu), \mu)+\gamma z(\mu)=x(\mu)$ and $j z(\mu)=y(\mu)$.

PROPOSITION 2 [1]. Let the conditions of Proposition 1 hold, let the $x(\mu): B \rightarrow B_{1}, y(\mu): B \rightarrow B_{2}$ be continuous and let $\left\|N_{i}(x, \mu)-N_{i}(y, \mu)\right\|_{i}<$ $M\|x-y\|_{i}(i=1,2)$, where $\gamma>M>0$.

Then the map $z(\mu): B \rightarrow B_{1}$, satisfying the equation $N_{1}(z(\mu), \mu)+\gamma z(\mu)=$ $x(\mu)$ is continuous.

PROPOSITION 3 [1]. Let the conditions of Proposition 1 hold and let $z_{i}(\mu),(i=1,2)$ satisfy the equalities $N_{1}\left(z_{i}(\mu), \mu\right)+\gamma z_{i}(\mu)=x_{i}(\mu)$, $j z_{i}(\mu)=y_{i}(\mu), N_{2}\left(y_{i}(\mu), \mu\right)+\gamma y_{i}(\mu)=j x_{i}(\mu)$. Then $\left\|z_{1}(\mu)-z_{2}(\mu)\right\|_{1} \leqslant$ $\frac{1}{\alpha}\left\|x_{1}(\mu)-x_{2}(\mu)\right\|_{1}$. 
We shall formulate the results of Kirk-Schoneberg [2] in order to apply them to our problem.

Let $X$ and $Y$ be complete metric spaces. Recall that: 1) $Y$ is said to be metrically convex (Menger [6]) if for $a l 1$, $u$, ve $Y$ with $u \neq v$ there exists we $Y, w \neq u, w \neq v$, such that $\rho(u, v)=\rho(u, w)+\rho(w, v) ; 2)$ a mapping $T: X \rightarrow Y$ is said to be closed if $u_{n} \rightarrow$ ue $X$ and $T\left(u_{n}\right) \rightarrow z e Y$ imply $T(u)=z$.

LEMMA [2]. Let $T: X \rightarrow Y$ be a closed mapping. Fix ye Y. Let $U$ be an open subset of $X$ for which $T(U)$ is open in $Y$, and suppose

$$
\rho(T u, T v)>\rho(u, v)
$$

for all u,veclU. Suppose also that there exists $u_{0} e U$ such that

$$
\rho\left(T u_{0}, y\right)<\rho(T u, y)+\rho\left(T u_{0}, T u\right)
$$

for all ue aU.

Then there exists ue $U$ such that $T u=y$.

If $T$ is a mapping of $X$ into $Y$ and $D$ is an open subset of $X$, then $T$ is said to be locally expansive on $D$ if for all $u_{0} \in D$ there is a neighbourhood $N$ of $u_{0}$ contained in $D$ such that $\rho(T u, T v)>\rho(u, v)$ for all $\mathrm{u}, \mathrm{v} \in \mathrm{N}$.

PROPOSITION 4 [2]. Let $D C X$ be open, and suppose $T: X+Y$ is a closed mapping which is locally expansive on $D$. Suppose also that $T$ maps open subsets of $D$ on to open subsets of $Y$, and there exists $x_{0} \in D$ such that $\rho\left(T x_{0}, y\right)<\rho(T x, y)$ for all xe XID. Then ye $T(D)$.

COROLLARY [2]. Let $\mathrm{T}: \mathrm{X} \rightarrow \mathrm{Y}$ be a closed mapping, which maps open subsets of $X$ onto open subsets of $Y$. Suppose also that $T$ is locally expansive on all of $X$. Then $T(X)=Y$.

THEOREM 1. Let the following conditions hold:

1. the functions $\Delta_{i}(t), \tau_{1}(t): R_{+}^{1} \rightarrow R^{1}$ are measurable, $\tau_{1}(t)$ have the property (S) (Angelov, [1]) and $\int_{0}^{\infty}\left\|f\left(\tau_{1}(t)\right)\right\| d t<k \int_{-\infty}^{\infty}\|f(t)\| d t$ for some $k=$ const $>0$ and every fe $L^{1}\left(R^{1} ; B\right)$.

2. the function $X\left(\mu, t, u_{1}, \ldots, u_{m}, v_{1}, \ldots, v_{n}\right): B \times R_{+}^{1} \times B^{m+n}+B$ satisfies the Caratheodory condition and

$$
\begin{aligned}
& \left\|X\left(\mu, t, u_{1}, \ldots, u_{m}, v_{1}, \ldots, v_{n}\right)\right\|<\frac{1}{\gamma}\left[\alpha\left(\mu, t,\left\|u_{1}\right\|, \ldots,\left\|u_{m}\right\|\right)+\alpha_{o}{\left.\underset{1=1}{n}\left\|v_{1}\right\|\right]}_{\left\|X\left(\mu, t, u_{1}, \ldots, u_{m}, v_{1}, \ldots, v_{n}\right)-X\left(\mu, t, \bar{u}_{1}, \ldots, \bar{u}_{m}, \bar{v}_{1}, \ldots, \bar{v}_{n}\right)\right\|<}\right. \\
& \quad \frac{m}{r}\left[\beta\left(\mu, t,\left\|u_{1}-\bar{u}_{1}\right\|, \ldots,\left\|u_{m}-\bar{u}_{m}\right\|\right)+\sum_{1=1}^{n} \beta_{1}\left\|v_{1}-\bar{v}_{1}\right\|\right]
\end{aligned}
$$


for some constants $\gamma>\mathrm{m}>0, \alpha_{0}, \beta_{1}>0 ; \alpha, \beta: B \times \mathrm{R}_{+}^{\mathrm{m}+1} \rightarrow \mathrm{R}_{+}^{1}$ are comparison functions (Angelov $[1]) ; \alpha\left(\mu, ., u_{1}, \ldots, u_{m}\right), B\left(\mu, ., u_{1}, \ldots, u_{m}\right) \in L^{1}\left(R_{+}^{1}\right)$ and

$\int_{0}^{\infty} B(\mu, t, v, \ldots, v) d t+k v \underset{1=1}{n} \beta_{1}<v, v \in R_{+}^{1}$.

Besides, $X(\mu, t, \ldots)$ is uniformly continuous in $\mu$ with respect to the other variables (Angelov [1]).

3. the initial function $\varphi(., \mu) \in L^{1}\left(R_{-}^{1} ; B\right)$ for every $\mu \in B$ and

$$
\lim _{\mu \rightarrow \mu_{0}} \int_{-\infty}^{0}\left\|\varphi(t, \mu)-\varphi\left(t, \mu_{0}\right)\right\| d t=0 .
$$

Then there exists a unique solution $x(., \mu) \in L^{1}\left(R^{1} ; B\right)$ of the initial value problem (2), which depends continuously on $\mu$.

Proof: Let $B_{1}$ be the Banach space $L^{1}\left(R^{1} ; B\right)$ with norm $\|f\|=\int_{-\infty}^{+\infty}\|f(t)\| d t$ and $B_{2}$ be the Banach space $L^{1}\left(R_{-}^{1} ; B\right)$ with norm $\|g\|_{2}=\int_{-\infty}^{0}\|g(t)\| d t$.

Define the operators $N_{i}: B_{i} \times B \rightarrow B_{i}(i=1,2)$

$N_{1}(f, \mu)(t)= \begin{cases}-\gamma X(\mu, t,[\Psi(0, \mu)+ & \Delta_{i}(t) \\ \left.\left[f\left(\tau_{1}(t)\right)\right]_{1=1}^{n}\right), & t>0 \\ 0 \quad, & t<0\end{cases}$

fe $B_{1}$, we $B$ and $N_{2}(g, \mu)(t)=0, t<0$, ge $B_{2}$, we $B$.

The map $j: B_{1} \rightarrow B_{2}$ is defined as in Theorem 1 (Angelov [1]).

Since $N_{1}(f, \mu)(t)$ is strongly measurable, then the inequalities

$\left\|N_{1}(f, \mu)(t)\right\|<\alpha\left(\mu, t,\|\Psi(0, \mu)\|+\|f\|_{1}, \ldots, \Psi(0, \mu)\|+\| f \|_{1}\right)$

$$
+a_{0} \sum_{1=1}^{n}\left\|f\left(\tau_{1}(t)\right)\right\|
$$

show that $f \in B_{1}$ implies $N_{1}(f, \mu) \in B_{1}$ for each $\mu \in B$.

We are going to show the Lipschitz continuity of the operator $\mathrm{N}_{1}$;

$$
\begin{aligned}
& \int_{0}^{\infty}\left\|N_{1}(f, \mu)(t)-N_{1}(g, \mu)(t)\right\| d t \leqslant m\left[\int_{0}^{\infty} \beta\left(\mu, t,\|f-g\|_{1}, \ldots,\|f-g\|_{1}\right) d t\right. \\
& \left.+\sum_{1=1}^{n} \beta_{1} \int_{0}^{\infty}\left\|f\left(\tau_{1}(t)\right)-g\left(\tau_{1}(t)\right)\right\| d t\right] \\
& <m\left[\int_{0}^{\infty} B\left(\mu, t,\|f-g\|_{1}, \ldots,\|f-g\|_{1}\right) d t+k\|f-g\|_{1} \underset{1=1}{\sum} \sum_{1}^{n}\right]<m\|f-g\|_{1} \text {. }
\end{aligned}
$$


Further on the proof is analogous to the one of Theorem 1 trom Angelov[1].

THEOREM 2. Let the conditions of Theorem 1 hold and 1 et $x\left(\varphi_{i}, \mu\right)(t)$, $(i=1,2)$ be solutions of the problem (2) with initial functions $\varphi_{i}(t, \mu)$. Then

$\int_{-\infty}^{\infty}\left\|x\left(\varphi_{1}, \mu\right)(t)-x\left(\varphi_{2}, \mu\right)(t)\right\| d t<\frac{\gamma}{\gamma-m} \int_{-\infty}^{0}\left\|\varphi_{1}(t, \mu)-\varphi_{2}(t, \mu)\right\| d t$.

THEOREM 3. Let the conditions of Theorem 1 hold true. If, in addition, we suppose:

1. $\psi(0,$.$) is locally expansive, closed and maps open subsets of B$ onto open subsets of $B$.

2. for every $\mu$ B $B$ there is a neighbourhood $U$ of $\mu$ such that

$\left\|X\left(\mu_{1}, t, \ldots\right)-X\left(\mu_{2}, t, \ldots\right)\right\| \geqslant \xi(t)\left\|_{\mu_{1}}-\mu_{2}\right\|$

for $\mu_{1}, \mu_{\infty} 2^{e U}$, where $\xi(t) \in L^{1}\left(R_{+}^{1}\right), \xi(t)>0$ and the mapping

$Q(\mu)=\int_{0} X\left(\mu, t, u_{1}, \ldots, v_{n}\right) d t$ is closed and maps open subsets of $B$ onto open subsets of $B$, provided the last integral exists.

Then there exists a value of the parameter $\mu_{0}$ such that $x\left(t, \mu_{0}\right)$ is a solution of the problem (2) and the solution $y\left(t, \mu_{0}\right)$ of (1) satisfies the condition $\lim _{t \rightarrow \infty} y\left(t, \mu_{0}\right)=y_{\infty}$.

Proof: Let $B{ }_{1}$ be the Banach space $L^{1}\left(R^{1} ; B\right)$ and $B_{2}$ be the Banach space $L^{1}\left(R_{-}^{1} ; B\right)$.

Define the operators $N_{i}: B_{i} \times B_{i}+B_{i}(i=1,2), T: B \times B_{1} \rightarrow B$ :

$N_{1}(f, \mu)(t)=\left\{\begin{array}{l}-\gamma X\left(\mu, t,\left[\Psi(0, \mu)+\Delta_{i}(t)\right.\right. \\ \left.\left[f\left(\tau_{1}(t)\right)\right]_{1=1}^{n}\right), t>0 \\ 0, t<0\end{array}\right.$

where fe $B_{1}, \mu \in B ; N_{2}(g, \mu)(t)=0, t<0, g \in B_{2}$, $\mu \in B$.

$$
\begin{gathered}
T(\mu, f)=y_{\infty}-\psi(0, \mu)-\int_{0}^{\infty} X\left(\mu, t,\left[\psi(0, \mu)+\int_{0}^{\Delta_{i}(t)} f(s) d s\right]_{i=1}^{m},\right. \\
\left.\left[f\left(\tau_{1}(t)\right)\right]_{1=1}^{n}\right) d t, f \in B_{1} .
\end{gathered}
$$

Proposition 1 implies an existence of a unique function $x(t, \mu)$ which satisfies (2) and depends continuously on $\mu$. 
On the other hand, it is easy to verify that the operator $T(\mu, f)$ satisfies all conditions of Corollary. Indeed, $T(\mu, f)$ is closed and maps open subsets of $B$ onto open subsets of $B$. Besides, for every ue $B$ there is a neighbourhood $U$ of $\mu$ such that

$\left\|T\left(\mu_{1}, f\right)-T\left(\mu_{2}, f\right)\right\| \geqslant c_{\psi}\left\|\mu_{1}-\mu_{2}\right\|+\int_{0}^{\infty} \xi(t) d t\left\|\mu_{1}-\mu_{2}\right\|$

for all $\mu_{1}, \mu_{2} \in U$. Consequently, there is $\mu_{0} \in B$ such that $T\left(\mu_{0}, f\right)=0$. So we obtain

$$
\begin{aligned}
& \lim _{t \rightarrow \infty} y\left(t, \mu_{0}\right)=\psi\left(0, \mu_{0}\right)+\int_{0}^{\infty} x\left(s, \mu_{0}\right) d s= \\
& \psi\left(0, \mu_{0}\right)+\sum_{0}^{\infty} x\left(\mu_{0}, s,\left[\psi\left(0, \mu_{0}\right)+\int_{0}^{\iota_{i}(s)} x\left(\theta, \mu_{0}\right) d \theta\right]_{i=1}^{m},\right. \\
& \left.\left[x\left(\tau_{1}(s)\right)\right]_{1=1}^{I}\right) d s=\psi\left(0, \mu_{0}\right)+y_{\infty}-\psi\left(0, \mu_{0}\right)=y_{\infty} .
\end{aligned}
$$

Theorem 3 is thus proved.

Finally we shall note that an analogous result can be formulated for systems of the type:

$$
\begin{aligned}
& y_{i}^{\prime}(t)=x_{i}\left(\mu_{i}, t,\left[y_{1}\left(\Delta_{1 k}(t)\right)\right]_{k=1}^{m_{1}}, \ldots,\left[y_{s}\left(\Delta_{s k}(t)\right)\right]_{k=1}^{m_{s}},\right. \\
& \left.\left[y_{1}^{\prime}\left(\tau \tau_{1 k}(t)\right)\right]_{k=1}^{n_{1}}, \ldots,\left[y_{s}^{\prime}\left(\tau{ }_{s k}(t)\right)\right]_{k=1}^{n_{s}}\right), t>0 \\
& y_{i}(t)=\psi_{i}\left(t, \mu_{i}\right), y_{i}^{\prime}(t)=\frac{\partial \psi_{i}\left(t, \mu_{i}\right)}{\partial t}, t<0 \\
& (i=1,2, \ldots, s) .
\end{aligned}
$$

\section{REFERENCES}

1. ANGELOV V.G. Some neutral equations with a control parameter. Bull. Austr. Math. Soc., 23, No 3 (1981), 383-394.

2. KIRK W.A., SCHÖNEBERG T. Mappings theorems for local expansions in metric and Banach spaces. J. Math. Ana1. App1., 72, No 1, (1979), 114-121.

3. HILLE E., PHILLIPS R. Functional Analysis and Semigroups, XXXI, Amer. Math. Soc., R I, Providence, 1957.

4. SEIDOV Z.B. Boundary value problem for differential equations with deviating argument. Differential Equations, v. 12, No 3 (1976), 562-566 (in Russian).

5. KANTOROVICH L.V., AKILOV G.P. Functional analysis, Nauka, Moscow 1977, (in Russian).

6. MENGER $\mathrm{K}$. Untersuchungen Uber allgemeine Metrik. Math. Ann. 100 (1928), 75-163. 


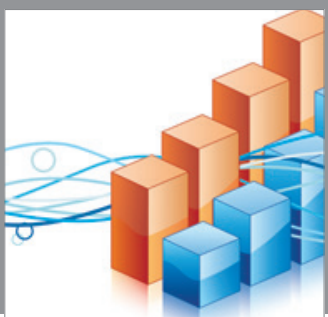

Advances in

Operations Research

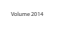

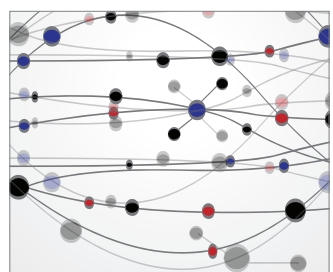

\section{The Scientific} World Journal
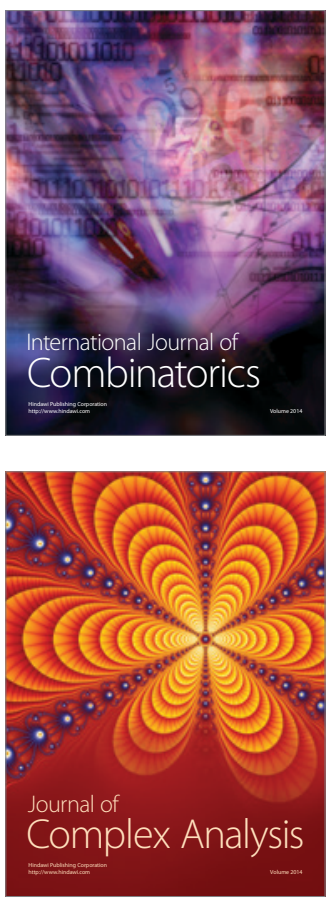

International Journal of

Mathematics and

Mathematical

Sciences
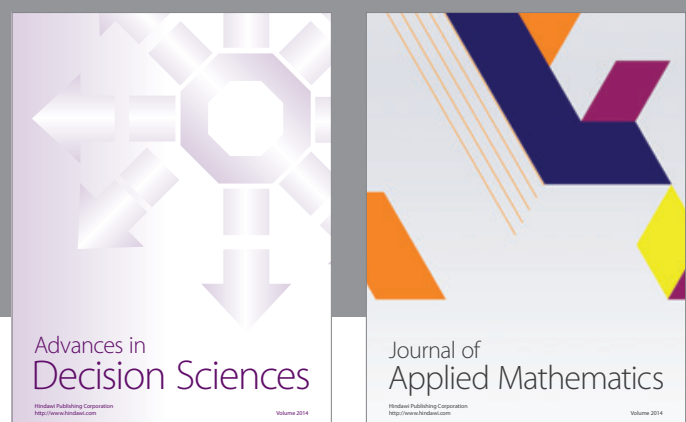

Journal of

Applied Mathematics
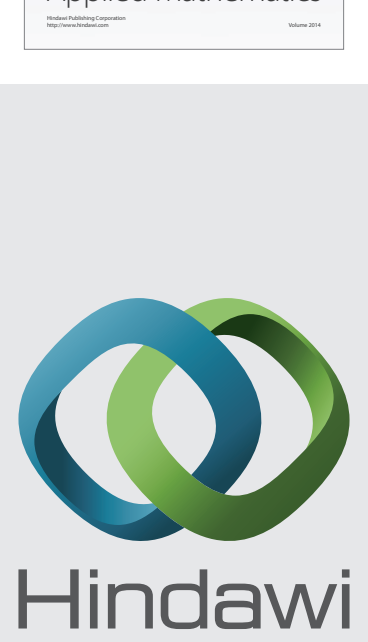

Submit your manuscripts at http://www.hindawi.com
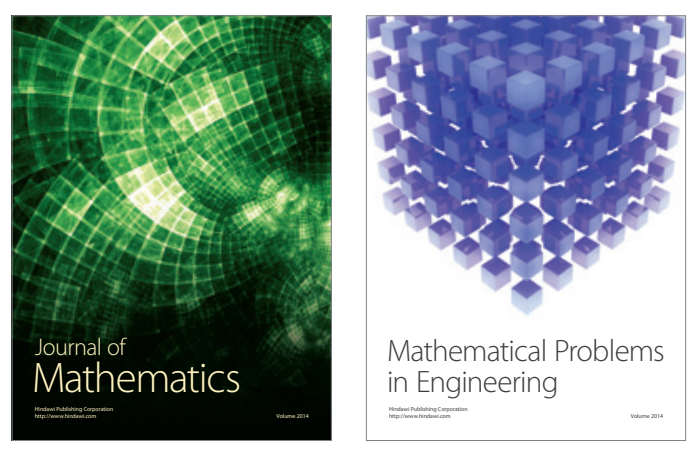

Mathematical Problems in Engineering
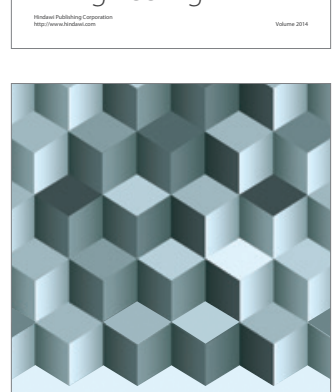

Journal of

Function Spaces
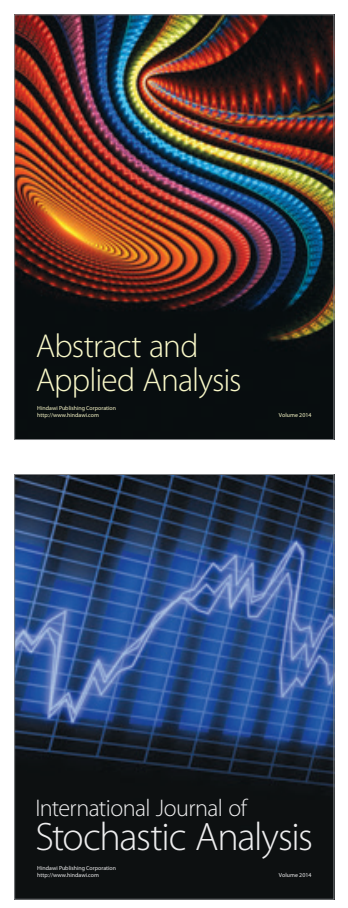

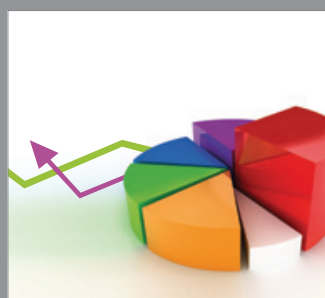

ournal of

Probability and Statistics

Promensencen
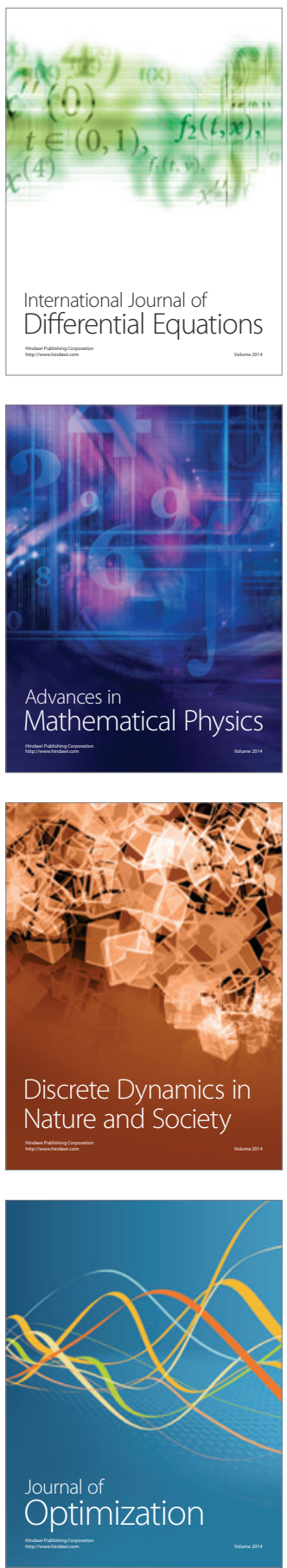